\title{
The relationship of dispositional compassion for others with depressive symptoms over a 15-year prospective follow-up
}

\begin{abstract}
Aino Saarinen ${ }^{1,2}$, Liisa Keltikangas-Järvinen ${ }^{2}$, C. Robert Cloninger ${ }^{3}$, Juha Veijola ${ }^{4}$, Marko Elovainio ${ }^{2,5}$, Terho Lehtimäki ${ }^{6}$, Olli Raitakari ${ }^{7}$, Mirka Hintsanen ${ }^{1, *}$
\end{abstract}

${ }^{1}$ Research Unit of Psychology, University of Oulu, Finland

${ }^{2}$ Department of Psychology and Logopedics, Faculty of Medicine, University of Helsinki, Finland

${ }^{3}$ Department of Psychiatry, Washington University, St. Louis, United States

${ }^{4}$ Department of Psychiatry, Institute of Clinical Medicine, University of Oulu and Oulu University Hospital, Oulu, Finland

${ }^{5}$ National Institute for Health and Welfare, Helsinki, Finland

${ }^{6}$ Department of Clinical Chemistry, Fimlab Laboratories and Faculty of Medicine and Health Technology, Finnish Cardiovascular Research Center, Tampere University, Tampere, Finland

${ }^{7}$ Research Centre of Applied and Preventive Cardiovascular Medicine, University of Turku, Finland; Department of Clinical Physiology and Nuclear Medicine, Turku University Hospital, Finland

* Corresponding author: Mirka Hintsanen. Research Unit of Psychology, University of Oulu, Oulu, Finland. P.O. Box 2000 (Yliopistokatu 9), 90014 University of Oulu, Oulu, Finland. Email: mirka.hintsanen@oulu.fi; gsm: +358 505695243 
Dispositional compassion and depressive symptoms

\section{Abstract}

Background: The aim of this study was to investigate (i) the direction of the relationships between dispositional compassion for others and depressive symptoms over a 15-year follow-up in adulthood and (ii) the longitudinal associations of dispositional compassion with total depressive symptoms and various depressive subsymptoms (i.e. negative attitude, performance difficulties, and somatic complaints) from early adulthood to middle age. Methods: The participants $(N=1676)$ came from the prospective Young Finns Study. Dispositional compassion was assessed with the Temperament and Character Inventory and depressive symptoms with a modified version of the Beck Depression Inventory. The measurements were conducted between 1997-2012 including three measurement points. The data was analyzed using structural equation models and multilevel models for longitudinal design. Results: The predictive relationships were more likely to proceed from high dispositional compassion for others to lower depressive symptoms than in the opposite direction. Additionally, high dispositional compassion predicted a lower total score of depressive symptoms and also lower scores of various depressive subsymptoms (negative attitude, performance difficulties, somatic complaints) in early adulthood. These associations, however, weakened over years and became non-significant in middle age. All the findings were sustained after controlling for age, gender, and socioeconomic factors in childhood and adulthood. Limitations: Depressive symptoms were mostly mild and non-clinical in our sample. The findings cannot be directly generalized to severe depressive symptomatology.

Conclusions: When tailoring psychiatric interventions, it is necessary to be aware that compassion for others may lower the risk for the onset and maintenance of depressive symptoms, especially in early adulthood. 


\section{Introduction}

It has been estimated that minor or subclinical depression occur in as many as $12-17 \%$ of the population (Fils et al., 2010; Goldney et al., 2004). Those individuals have a substantially increased risk for developing a major depressive disorder later in their life (Cuijpers et al., 2004). Hence, there has been a growing interest for developing early preventive treatments for minor depression. Previously, a meta-analysis concluded that anti-depressive medications seem not to alleviate minor depression effectively (e.g. Barbui et al., 2011).

Regarding psychosocial factors, it is known that there exist a variety of risk factors increasing the vulnerability to depression, such as anxiety, substance abuse, insecure attachment, early adversities (e.g. Bifulco et al., 2002; Harald-Gordon et al., 2012). Recently, however, there has been a growing interest for protective factors that might prevent the course from mild to severe depression. In this context, there is promising evidence for positive psychology interventions as initial treatments for mild depression (e.g. van Zoonen et al., 2014). In particular, compassion and taking care of others are suggested to effectively reduce shame and self-criticism (Gilbert, 2014, 2019). Further, intervention studies have shown that practicing compassion predicts reductions in depressive symptoms (Braehler et al., 2013; Gilbert \& Procter, 2006; Judge et al., 2012; Leaviss \& Uttley, 2015; Lucre \& Corten, 2013).

Still, research on long-term associations between dispositional compassion and depression are missing. Hence, more precise knowledge of the link of compassion with depressive symptoms might provide new tools for tailoring early interventions for mild depression in order to prevent the onset of a major depressive disorder.

Compassion is defined as a disposition to feel concern for other's suffering that is followed by the desire to alleviate the suffering and improve other's well-being (Goetz et al., 2010). 
Compassion includes two necessary components: an affective component (recognizing other's emotional distress and tolerating it) and a motivational component (motivation to take the appropriate actions to relieve other's negative affective state) (Gilbert, 2017; Gilbert \& Mascaro, 2017). Compassion can be manifested both as temporary compassionate feelings for others that may appear and disappear in accord with situational factors (i.e. compassionate states) or as a more stable trait that endures over time (i.e. dispositional compassion) (Goetz et al., 2010).

Previous research has demonstrated that high dispositional compassion for others is related to lower depressive symptoms (e.g. Balsamo et al., 2013; Richter et al., 2000). However, the current evidence is mostly based on cross-sectional studies. To our knowledge, the longest follow-up in studies investigating the link of compassion to depressive symptoms has been only 12 months (Corruble et al., 2002). Hence, it has remained unclear whether dispositional compassion is associated with depression steadily over age in adulthood or whether age-related changes in this association might occur. Consequently, there has been a strong demand for studies with long-term follow-ups that investigate the relationship of compassion-related concepts and depressive symptoms (e.g. Burnette et al. 2009; Cusi et al., 2011; Schreiter et al., 2013).

Because of the lack of studies with long-term follow-ups and several measurement points, the direction of the relationship between compassion and depressive symptoms have remained largely unclear (Celikel et al., 2010; Cusi et al., 2011; Farmer et al., 2003; Schreiter et al., 2013; Tully et al., 2016). It has been previously suggested that depressive states may cause temporary changes in personality traits (Hirschfeld, 1999; Hur \& Kim, 2009). For example, certain compassion-related traits are found to decrease along with the progression of a depressive disorder (e.g. Cusi et al., 2011). These changes in personality traits are suggested to have an adaptive and compensatory role during depressive episodes (Hansenne et al., 1999): for example, lowered compassion for others might enhance caretaking of oneself during an acute depressive state. Additionally, it has been found that depressed 
individuals commonly have transient deficits in social cognition and executive functions, such as cognitive flexibility or directing attention, that in turn are related to difficulties in taking others' perspectives (e.g. Eslinger, 1998; McKinnon and Moscovitch, 2007; Schreiter et al., 2013).

On the other hand, the relationship may proceed in the opposite direction, i.e. low dispositional compassion might predispose to the development of depressive symptoms. Social intolerance, revengefulness, and difficulties in perspective taking (i.e. indicators of low compassion) may lead to negatively biased interpretations of social situations and eventually result in lowered mood (Balsamo et al., 2013; Cusi et al., 2011; Hur \& Kim, 2009). Furthermore, in accordance with the social mentality theory, such psychobiological systems that facilitate one's willingness to caregiving of others provide the basis for one's self-representation (Liotti \& Gilbert, 2011).

There exist also a range of psychophysiological mechanisms that may mediate the path from compassion to depression. Compassion-practicing and loving-kindness interventions are related to better coping with stress, for example, lower cortisol levels and weaker inflammatory reactions (e.g. some cytokines) when facing psychosocial stressors (Abelson et al., 2014; Pace et al., 2009). Lower level of inflammation, in turn, is found to decrease the risk for sickness behaviors such as changes in appetite or sleep (Dantzer, 2001) and depression (Dantzer et al., 2008; van den Biggelaar et al., 2007). Additionally, compassionate states are related to more favorable heart rate variability, such as higher respiratory sinus arrhythmia, and lower heart rate (Stellar et al., 2016) that are linked to lower depression (Berntson \& Cacioppo, 2004).

Moreover, there is accumulating evidence about the relationship between compassion and neurophysiological processes in the brain (see Seppälä et al., 2017). That is, practicing compassion is related to functional changes in such neural circuitries that contribute to attuning to others' affective states (e.g. amygdala, inferior parietal cortex, nucleus accumbens) (Valk et al., 2017; Weng et al., 2013, 2018). These brain networks, in turn, are shown to be related to depression (e.g. Fitzgerald et al., 2008). 
Finally, compassionate states and caring behavior correlate with oxytocin-related genes (Gilbert, 2019; Kogan et al., 2010) and also with the activity of hypothalamus (Immordino-Yang et al., 2009) that plays a role in oxytocin hormone production. Oxytocin production, in turn, is firmly linked to depression (McQuaid et al., 2014). Taken together, this evidence implies that individuals with low dispositional compassion may be more susceptible for experiencing stress, having less favorable health behavior and cardiovascular health, and having less adaptive functioning in depression-related brain networks that all increase their risk for depressive symptoms.

The first aim of the present study was to investigate the direction of the relationships between depressive symptoms and dispositional compassion for others in adulthood, i.e. whether depressive symptoms are more likely to lower the level of dispositional compassion for others or whether high dispositional compassion might protect against the development of depressive symptoms. The second aim was to investigate the longitudinal associations of dispositional compassion for others with total depressive symptoms from early adulthood to middle age. There is accumulating evidence that individuals with depressive disorders include a highly heterogeneous population (Rantala et al., 2017). Thus, it has been emphasized that various depressive symptoms should be investigated separately (Fried \& Nesse, 2005). Hence, the current study investigates the relationship of dispositional compassion with various depressive subsymptoms (i.e. negative attitude, performance difficulties, and somatic complaints). We used data from the Young Finns Study that included a 15-year prospective follow-up and several measurement points.

\section{Methods}

\subsection{Participants}

The participants were members of the prospective Young Finns Study. The participants were selected 
randomly from six birth cohorts (born in 1962, 1965, 1968, 1971, 1974, and 1977) from the population register of the Social Insurance Institution. The original sample included 3596 participants at the baseline measurement in 1980. Since then, eight follow-up measurements have been conducted between 1983 and 2012 (when participants were aged 35-50 years). For the present study, dispositional compassion and depressive symptoms were assessed in 1997, 2001, and 2012; parental socioeconomic factors in 1980; and participants' socioeconomic factors in 2011. We included in the analyses all the participants with data on dispositional compassion, depressive symptoms, and socioeconomic factors in childhood and adulthood in at least one of the measurement times (e.g. data on compassion in 1997, 2001, or 2012). The final data included 1,676 participants.

The Young Finns Study was carried out in accordance with the Declaration of Helsinki. Additionally, all the Finnish universities with medical schools approved the study design. Before participation, all the participants or their parents (for participants aged below 12 years) provided written informed consent after the nature of the procedures had been fully explained. The design and methods of the Young Finns Study is described more precisely elsewhere (Raitakari et al., 2008).

\subsection{Measures}

Dispositional compassion was evaluated with the version 9 of the Temperament and Character Inventory (TCI, Cloninger, Przybeck, Svrakic, \& Wetzel, 1994). In the TCI, dispositional compassion is a subscale of the character dimension Cooperativeness. The scale of dispositional compassion $(\alpha=.86-.87$ in 1997,2001 , and 2012) consists of 10 self-rating statements (e.g. "It gives me pleasure to help others, even if they have treated me badly"). The statements are rated using a 5-point Likert-scale ranging from 1 (completely disagree) to 5 (completely agree). For each measurement time, the total score of dispositional compassion was calculated for all the participants who had data on at least $50 \%$ 
of the items of the scale. The total scores of dispositional compassion were standardized with the year 1997 (i.e. the first measurement year) mean and standard deviation.

The convergent and discriminant validity of the dispositional compassion scale have been confirmed previously. Specifically, high values of the compassion scale are found to correlate with higher social warmth, altruistic behavior, sociability, and positive emotionality (García et al., 2012; Schmidt et al., 2003). Instead, low values of the compassion scale are related to higher hostility and aggression (García et al., 2012; Lee et al., 2012) and also higher narcissistic (De Fruyt et al., 2006) and psychopathic features (Paal \& Bereczkei, 2007). Additionally, the test-retest reliability of the scale is found to be high (e.g. Hansenne et al, 2005). In our sample, the test-retest correlations between the measurement years were as follows: between 1997-2001 $r=.67$; between 2001-2012 $r=.69$; between $1997-2012 r=.59$.

Depressive symptoms were assessed with a modified version of the Beck Depression Inventory. The modified BDI (mBDI) includes the second mildest statements of the BDI (i.e. the statements that represented the second mildest depressive symptoms in the items of the BDI). The statements are answered with a 5-point Likert-scale ranging from 1 (totally disagree) to 5 (totally agree) $(\alpha=.91-.93$ in 1997, 2001, and 2012). The mBDI includes 3 factors: negative attitude $(\alpha=.78-.84$ in 1997, 2001, and 2012), performance difficulties $(\alpha=.85-.88)$, and somatic complaints $(\alpha=.69-.74)$. The mBDI is described more precisely elsewhere (Elovainio et al., 2005). Compared to the original BDI-II, the mBDI is found to capture mild and subclinical depressive symptoms more sensitively (e.g. Katainen et al., 1999; Nurmi et al., 1995; Rosenström et al., 2012). For each measurement time, we calculated a total score of depressive symptoms and various depressive subsymptoms (i.e. negative attitude, performance difficulties, and somatic complaints) for all the participants who had data available on at least $50 \%$ of the items. The scores of depressive symptoms were standardized with the 
year 1997 (i.e. the first measurement year) mean and standard deviation.

Participants' and their parents' socioeconomic factors included education level and annual income. As has been done also previously (Pulkki-Råback et al., 2015), parental educational level was classified into 3 categories $(1=$ comprehensive school, i.e. the first 9 years of school; $2=$ high school or occupational school; $3=$ academic, i.e. university or college). Parental annual income included 8 categories ( $1=$ less than 15000 Finnish mark; 8=more than 100000 Finnish mark). Participants' educational level was assessed using the number of educational years (ranging from 8 to 34). Participants' annual income was assessed with a 13-point scale (1=less than $5000 €$ per year; $13=$ more than $60000 €$ per year). We added the socioeconomic factors to the analyses as separate variables. Participants' and their parents' level of income and the number of participants' educational years were standardized (mean $=0$, standard deviation $=1)$.

\subsection{Statistical analyses}

We conducted statistical analyses using the version 13.0 of Stata SE. We investigated attrition by comparing the study variables between the included $(N=1676)$ and excluded $(N=1920)$ participants. The direction of the relationships between dispositional compassion and depressive symptoms were examined using structural equation models with cross-lagged panel design (MLMV estimation). We conducted altogether 4 models: (1) an autoregressive model including only stability coefficients (the variables at each time point were predicted by the same variables at the previous time point) and covariances between dispositional compassion and depressive symptoms at each timepoint; (2) a model that included also the cross-lagged predictive coefficients from dispositional compassion at each time point to depressive symptoms at the next timepoint; (3) a model that included the cross-lagged predictive coefficients in the opposite direction (i.e. from depressive symptoms at each time point to 
dispositional compassion at the next timepoint); and (4) a model with the cross-lagged predictive coefficients in both directions between dispositional compassion and depressive symptoms. In the models, we included the scores of various depressive subsymptoms (negative attitude, performance difficulties, somatic complaints) because it has been suggested that various depressive symptoms should be investigated separately (Fried \& Nesse, 2005).

We assessed the statistical fit of the models $1-4$ by examining the values of the Comparative Fit Index (CFI), the Root-Mean-Square Error of Approximation (RMSEA), and the Bayesian Information Criterion (BIC). Previously, it has been found that adequate values of the CFI range over 0.95, and the value of RMSEA should be below 0.06 (Hu \& Bentler, 1999). Moreover, lower scores of the $\chi^{2}$ test of absolute model fit and the BIC refer to better model-fit (Schreiber et al., 2006).

The longitudinal associations of dispositional compassion with depressive symptoms (measured with the mBDI) were investigated using multilevel models for repeated measurements with maximum likelihood estimation. The multilevel models derive estimates for fixed effects (classic regression coefficients) and random effects (between-individual variation in intercept and slopes). We estimated separate growth curve trajectories for the total score of depressive symptoms and for the scores of various depressive subsymptoms (i.e. negative attitude, performance difficulties, and somatic complaints). Intercept, age, age-squared, dispositional compassion and its age-interactions, gender, and socioeconomic factors in childhood and adulthood were set as fixed effects. Intercept and age were treated also as random effects. Age referred to participant's age in years at each measurement time and ranged from 20 (the age of the youngest study cohort in 1997) to 50 (the age of the oldest study cohort in 2012). In order to reduce potential multicollinearity in the multilevel models, age was centered at age 20. 
Dispositional compassion and depressive symptoms

\section{Results}

The means, standard deviations, and frequencies of the study variables are shown in Table 1. Attrition analyses revealed that included participants were slightly older (35.69 vs. 35.23, p<.01) than excluded participants. Women were more likely to participate than men $(51.5 \%$ vs. $41.6 \%, \mathrm{p}<.001)$.

Additionally, included participants had slightly higher dispositional compassion (3.69 vs. 3.58, p<.001) and a lower score of total depressive symptoms (2.06 vs. 2.16, $\mathrm{p}<.001)$, depression-related negative attitude (2.08 vs. $2.22, \mathrm{p}<.001)$ and performance difficulties (1.99 vs. $2.10, \mathrm{p}<.001)$ than excluded participants. There was no attrition bias in depression-related somatic complaints. Regarding socioeconomic factors, included participants had a higher number of educational years (15.53 vs. 14.33, $\mathrm{p}<.001)$ and their parents had a higher level of income (4.91 vs. $4.68, \mathrm{p}<.001)$ and had less likely a low educational level $(32.3 \%$ vs. $36.8 \%, \mathrm{p}<.01)$, when compared to excluded participants and their parents. There was no attrition bias in the level of income between included and excluded participants.

[Table 1 about here]

Table 2 shows the results of the structural equation models investigating the temporal relationships between dispositional compassion and depressive symptoms. Model 1 (autoregressive model without cross-lagged predictive paths) had acceptable model fit (RMSEA=0.056, $\mathrm{CFI}=0.959, \mathrm{BIC}=64308.345)$. Nevertheless, model 2 (cross-lagged predictive coefficients from dispositional compassion to depressive symptoms) had significantly better model-fit than model $1\left[\chi^{2}(\operatorname{model} 2\right.$ vs. 1$)=45.908$, $\mathrm{p}<0.001]$. This indicated that there were predictive relationships between dispositional compassion and depressive symptoms, besides the associations of each trait with itself across time in an autoregressive model. Furthermore, model 2 had better model-fit than model 3 (cross-lagged predictive coefficients 
from depressive symptoms to dispositional compassion), when comparing the values of RMSEA (0.056 in model 2 vs. 0.058 in model 3), CFI (0.963 vs. 0.959), and BIC (64306.981 vs. 64346.727). Hence, the predictive relationships from dispositional compassion to depressive symptoms appeared to be stronger than in the opposite direction. Finally, adding cross-lagged predictive coefficients in both directions between dispositional compassion and depressive symptoms (in model 4) did not improve the model-fit significantly $\left[\chi^{2}(\operatorname{model} 4\right.$ vs. 2$\left.\left.)=6.536, p=0.366\right)\right]$. Taken together, model 2 had better model-fit than models 1,3 , and 4 . Hence, the predictive pathways were more likely to proceed from dispositional compassion to depressive symptoms than vice versa. The model 2 is illustrated in Figure 1.

[Table 2 about here]

[Figure 1 about here]

Table 3 presents the results of the multilevel models related to the longitudinal associations of dispositional compassion with depressive symptoms. When predicting the total score of depressive symptoms, it was found that high dispositional compassion was associated with lower depressive symptoms in adulthood. However, as indicated by the negative age-interaction effect, this association was slightly weakened over age and became non-significant in middle age. When predicting various depressive subsymptoms, it was found that high dispositional compassion predicted lower depressionrelated negative attitude, performance difficulties, and somatic complaints. The associations of dispositional compassion with depression-related performance difficulties and somatic complaints became weaker over age, as indicated by the negative age-interaction effects. Taken together, the 
association of high dispositional compassion with total score of depressive symptoms and various depressive subsymptoms appeared to be stronger in early adulthood and become slightly weaker over age. The results of the multilevel models are illustrated in Figures $2 \mathrm{a}-\mathrm{d}$.

As additional analyses, we investigated whether there might exist a threshold effect: whether average level of compassion might reach a critical level in middle age, so that increases in the level of compassion might not have any additional protective effect against depression. Hence, we standardized the values of dispositional compassion in 1997, 2001, and 2012 with the year 1997 mean and standard deviation. That is, the scale of compassion was changed to the scale of the first measurement year. All the significant associations remained the same. The associations of dispositional compassion with depressive symptoms still weakened over age and became non-significant in middle age.

[Table 3 about here]

[Figure 2 about here]

\section{Discussion}

To our knowledge, this was the first study to investigate the relationship of dispositional compassion for others with depressive symptoms over a long-term prospective follow-up with several measurement points. The findings showed that the direction of the relationship was more likely to proceed from dispositional compassion to depressive symptoms than in the opposite direction. That is, high dispositional compassion for others appeared to have a protective effect against the development of 
depressive symptoms, but depressive symptoms seemed not to decrease the level of dispositional compassion for others at similar extent. Additionally, we found that high dispositional compassion was related to lower total depressive symptoms and also with lower scores of various depressive subsymptoms (i.e. depression-related negative attitude, performance difficulties, and somatic complaints) in early adulthood. However, the associations of dispositional compassion with depressive symptoms slightly weakened over age in adulthood and became non-significant in middle age. All these findings were sustained after taking into account age, gender, and socioeconomic factors in childhood and adulthood.

Previously, it has been suggested that while depressed individuals commonly feel shame or inferiority to others due to failures in e.g. occupational achievements, depression may not result in feelings of inferiority in the context of care-taking behavior or feeling concern for other's suffering (McEwan, Gilbert, \& Duarte, 2012). In line with this, our findings suggested that depressive symptoms may not predict lowered levels of compassion. Instead, our findings suggested that high dispositional compassion for others appeared to have a protective effect against the development of depressive symptoms.

Our findings showed that high compassion for others was associated with lower depression-related negative attitude (i.e. sadness) and also performance difficulties (i.e. social isolation, difficulties in concentration and decision-making, lowered level of functioning). It may be that abilities for social perspective taking and tolerance toward others prevent negatively biased interpretations of interpersonal situations and lower the risk for depressive mood (Balsamo et al., 2013; Cusi et al., 2011; Hur \& Kim, 2009). Additionally, as compassion includes a motivational component to alleviate others' suffering (Gilbert, 2017), high compassion is strongly linked to higher prosocial behavior and kindness toward others (e.g. Goetz et al., 2010). Practicing prosocial behavior, in turn, is related to higher social connectedness (e.g. Hutcherson et al., 2008) that buffers against depression (Rantala et al., 2018). Over 
the evolution, separation from the social network has been a substantial threat for the life (Rantala et al., 2018). Moreover, it is known that depression-related deficits in concentration or decision-making partly derive from depression-related negative rumination and challenges to detach from an egocentric viewpoint (e.g. Gotlib \& Joormann, 2010). High dispositional compassion for others is by definition contrary to egocentric thinking and, thus, may reduce the risk of some depression-related cognitive deficits.

Interestingly, the findings also revealed that high dispositional compassion for others was also related to lower depression-related somatic complaints (e.g. disturbances in sleep or appetite, increased fatigue, physical pains). Previously, it has been suggested that high compassion for others may be related to higher awareness of emotional states and bodily sensations and, in that way, protect against somatization symptoms (Clarke, 2016; Cloninger \& Docuku, 2016). Additionally, compassion for others is also linked to such physiological response patterns of parasympathetic nervous system and immune system that lower the risk for somatic complaints. For example, compassion training is related to lower cortisol levels and weaker inflammatory reactions when encountering psychosocial stressors (Abelson et al., 2014; Pace et al., 2009). Additionally, compassion is related to more favorable health behavior (Dantzer, 2001; Gluschkoff et al., 2018) and higher heart rate variability (Stellar et al., 2016). These physiological correlates, in turn, are shown to decrease the risk for depression (Berntson \& Cacioppo, 2004; Fitzgerald et al., 2008; McQuaid et al., 2014; Rantala et al., 2017).

Our results suggested that the link of compassion with depressive symptoms was stronger in early adulthood but became non-significant in middle age. In early adulthood, the most essential developmental tasks are strongly related to social functioning, for example, the formation of a romantic relationship or social integration in a working environment (Roisman et al., 2004). Encountering stressful life situations that are not in line with developmental tasks are known to be a strong risk factor 
for depression (Rantala et al., 2017). High dispositional compassion is related to better quality of social functioning (Goetz et al., 2010) and, thus, may promote the normative psychosocial development. By middle age, the social networks have commonly been established and new life tasks take place (Sheldon \& Kasser, 2001). For example, individuals may question whether they have chosen meaningful roles in their life. Especially, it has been shown that the need for self-actualization and spirituality significantly increases from middle age onwards (Ivtzan et al., 2013; Wink \& Dillon, 2002). Hence, it may be that among individuals with high compassion, the level of self-actualization or selftranscendence needs to increase in middle age to protect against depressive symptoms. Additionally, in middle age, one commonly encounters a variety of novel environmental factors such as aging-related diseases or deaths of close relatives. These environmental factors may increase the population-level incidence of depressive symptoms and, simultaneously, diminish the role of compassion or other personality characteristics for the onset of depression.

On the other hand, since compassion was found to slightly increase over age, the findings might also be explained by a threshold effect. That is, average level of compassion might reach a critical level in middle age, so that increases in the level of compassion might not have any additional protective effect against depression. However, this may not fully explain the findings. In our additional analyses, the values of dispositional compassion in 2001 and 2012 were scaled with the year 1997 level of compassion, and all the associations remained.

With regard to the limitations of our study, it must be taken into consideration that the level of depressive symptoms was mostly mild and non-clinical in our sample. Hence, our findings cannot be directly generalized to populations with severe and clinically significant depressive symptomatology. Nevertheless, a recent review highlighted that more research should be directed at understanding subclinical depression, since it strongly predicts later clinical depression and could provide new tools for tailoring early and preventive interventions (Lee et al., 2018). This was the 
primary aim of the present study. Therefore, we also used a modified version of the BDI that is found to capture especially effectively subclinical depressive symptoms in the general population (e.g. Katainen et al., 1999; Rosenström et al., 2012).

The present study had also several substantial strengths. Firstly, we had a prospective 15year follow-up with several measurement points of dispositional compassion and depressive symptoms. This enabled us to investigate the relationship of dispositional compassion with depressive symptoms from early adulthood to middle age and to disentangle whether the temporal pathways might run from depression to compassion or vice versa. This kind of prospective study has been widely called for previously (e.g. Celikel et al., 2010; Cusi et al., 2011; Farmer et al., 2003; Schreiter et al., 2013; Tully et al., 2016). Secondly, we had a comparatively large population-based sample that likely represented the general population with regard to most of its characteristics. Thirdly, we could control for several potential confounding factors, such as age, gender, and socioeconomic factors in childhood and adulthood. Finally, we used such questionnaires of dispositional compassion and depressive symptoms that have been used also previously and are shown to have high reliability (e.g. Gluschkoff et al., 2018; Hansenne et al., 2005; Katainen et al., 1999; Nurmi et al., 1995).

In clinical practice, it is important to be aware that high dispositional compassion for others may have a protective effect against the development of depressive symptoms, whereas depressive symptoms may not decrease the level of dispositional compassion. Hence, our findings are in line with a previous review (Leaviss \& Uttley, 2015) suggesting that increasing compassion through interventions might potentially lower the risk for the onset and maintenance of depressive symptoms. Moreover, the role of dispositional compassion for depressive symptoms may be especially strong among patients in their early adulthood, but weaker among middle-aged patients. When tailoring interventions for depressive patients, it is necessary to be aware that dispositional compassion may not 
only lower the risk of the core symptoms of depression (e.g. sadness, pessimism, and guilty), but also with depression-related performance difficulties and somatic complaints.

At a more practical level, compassion training for depressive patients could include both affective and motivational elements. Affective elements refer to practices how to shift attention from negative self-monitoring to recognizing others' emotional states (Kirby \& Gilbert, 2017). Further, depressive patients could have behavioral rehearsals to better tolerate other's suffering and to create a non-judgemental attitude toward others' negative affects (Kirby \& Gilbert, 2017). Importantly, however, some clinically depressed patients may not be able to directly attain compassionate feelings for their close others and, hence, they may experience failure during these affect-related practices (Gilbert, 2019). In those cases, it may be better to begin with the motivational components (Gilbert, 2019). Motivational components aim to increase depressed patients' abilities to find practical means to alleviate others' suffering. A variety of cognitive-behavioral interventions can be used to increase patients' awareness about the link between their care-taking behavior and others' affective states (Kirby \& Gilbert, 2017). Taken together, via these compassion-related practices depressive patients can shift their attention from negative self-rumination to positive care-taking behavior.

\section{Contributors}

A.S. and M.H. designed and executed the study and drafted the manuscript. A.S. analyzed the data. M.H. assisted with the data analyses. A.S., M.H., L.K.-J., C.R.C., J.V., M.E., T.L., and O.R. contributed to the interpretation of the results and collaborated with writing the manuscript. L.K.-J., M.H., T.L., and O.R. contributed to the data collection.

\section{Acknowledgements}


This study was supported financially by the Academy of Finland (M.H., grant number 308676). The Young Finns Study has been financially supported by the Academy of Finland: grants 286284, 134309 (Eye), 126925, 121584, 124282, 129378 (Salve), 117787 (Gendi), and 41071 (Skidi); the Social Insurance Institution of Finland; Competitive State Research Financing of the Expert Responsibility area of Kuopio, Tampere and Turku University Hospitals (grant X51001); Juho Vainio Foundation; Paavo Nurmi Foundation; Finnish Foundation for Cardiovascular Research ; Finnish Cultural Foundation; The Sigrid Juselius Foundation; Tampere Tuberculosis Foundation; Emil Aaltonen Foundation; Yrjö Jahnsson Foundation; Signe and Ane Gyllenberg Foundation (T.L); Diabetes Research Foundation of Finnish Diabetes Association; and EU Horizon 2020 (grant 755320 for TAXINOMISIS); and European Research Council (grant 742927 for MULTIEPIGEN project); Tampere University Hospital Supporting Foundation.

\section{Role of the funding source}

The funding source had no role in study design, data collection, data analysis, data interpretation, writing of the report, or in the decision to submit the article for publication.

\section{Conflict of interest}

None.

\section{References}

Abelson, J. L., Erickson, T. M., Mayer, S. E., Crocker, J., Briggs, H., Lopez-Duran, N. L., \& Liberzon, I. (2014). Brief cognitive intervention can modulate neuroendocrine stress responses to the Trier 
Social Stress Test: Buffering effects of a compassionate goal orientation.

Psychoneuroendocrinology, 44, 60-70.

Balsamo, M. (2013). Personality and depression: evidence of a possible mediating role for anger trait in the relationship between cooperativeness and depression. Comprehensive Psychiatry, 54, 46-52. https://doi.org/10.1016/j.comppsych.2012.05.007

Barbui, C., Cipriani, A., Patel, V., Ayuso-Mateos, J. L., \& van Ommeren, M. (2011). Efficacy of antidepressants and benzodiazepines in minor depression: systematic review and meta-analysis. The British Journal of Psychiatry, 198, 11-16. https://doi.org/10.1192/bjp.bp.109.076448

Berntson, G. G., \& Cacioppo, J. T. (2004). Heart rate variability: Stress and psychiatric conditions. Dynamic Electrocardiography, 57-64.

Bifulco, A., Moran, P. M., Ball, C., \& Bernazzani, O. (2002). Adult attachment style. I: Its relationship to clinical depression. Social Psychiatry and Psychiatric Epidemiology, 37, 50-59.

Braehler, C., Gumley, A., Harper, J., Wallace, S., Norrie, J., \& Gilbert, P. (2013). Exploring change processes in compassion focused therapy in psychosis: Results of a feasibility randomized controlled trial. British Journal of Clinical Psychology, 52, 199-214. https://doi.org/10.1111/bjc.12009

Burnette, J. L., Davis, D. E., Green, J. D., Worthington Jr, E. L., \& Bradfield, E. (2009). Insecure attachment and depressive symptoms: The mediating role of rumination, empathy, and forgiveness. Personality and Individual Differences, 46, 276-280. https://doi.org/10.1016/j.paid.2008.10.016

Clarke, D. D. (2016). Diagnosis and treatment of medically unexplained symptoms and chronic functional syndromes. Families, Systems, \& Health, 34, 309-316. http://dx.doi.org/10.1037/fsh0000228 
Cloninger, C. R., \& Dokucu, M. (2016). Somatizing and Dissociative Disorders. In The Medical Basis of Psychiatry (pp. 195-209). Springer, New York, NY.

Cloninger, C. R., Przybeck, T. R., \& Švrakić, D. M. (1994). The Temperament and Character Inventory (TCI): A guide to its development and use. St. Louis, MO: Center for Psychobiology of Personality, Washington University, pp. 19-28.

Corruble, E., Duret, C., Pelissolo, A., Falissard, B., \& Guelfi, J. D. (2002). Early and delayed personality changes associated with depression recovery? A one-year follow-up study. Psychiatry Research, 109, 17-25. https://doi.org/10.1016/S0165-1781(01)00366-3

Celikel, F. C., Kose, S., Erkorkmaz, U., Sayar, K., Cumurcu, B. E., \& Cloninger, C. R. (2010). Alexithymia and temperament and character model of personality in patients with major depressive disorder. Comprehensive Psychiatry, 51, 64-70. https://doi.org/10.1016/j.comppsych.2009.02.004

Cuijpers, P., de Graaf, R., \& van Dorsselaer, S. (2004). Minor depression: risk profiles, functional disability, health care use and risk of developing major depression. Journal of Affective Disorders, 79, 71-79. https://doi.org/10.1016/S0165-0327(02)00348-8

Cusi, A. M., MacQueen, G. M., Spreng, R. N., \& McKinnon, M. C. (2011). Altered empathic responding in major depressive disorder: relation to symptom severity, illness burden, and psychosocial outcome. Psychiatry Research, 188, 231-236.

https://doi.org/10.1016/j.psychres.2011.04.013

Dantzer, R. (2001). Cytokine-induced sickness behavior: where do we stand. Brain, Behavior, and Immunity, 15, 7-24. https://doi.org/10.1006/brbi.2000.0613

Dantzer, R., O'Connor, J. C., Freund, G. G., Johnson, R. W., \& Kelley, K. W. (2008). From inflammation to sickness and depression: when the immune system subjugates the brain. Nature Reviews Neuroscience, 9, 46-56. https://doi.org/10.1038/nrn2297 
De Fruyt, F., De Clercq, B. J., van de Wiele, L., \& Van Heeringen, K. (2006). The validity of Cloninger's Psychobiological Model versus the Five-Factor Model to predict DSM-IV personality disorders in a heterogeneous psychiatric sample: Domain facet and residualized descriptions. Journal of Personality, 74, 479-510. https://doi.org/10.1111/j.1467-6494.2006.00382.x

Elovainio, M., Keltikangas-Järvinen, L., Kivimäki, M., Pulkki, L., Puttonen, S., Heponiemi, T., ..., Raitakari, O.T., 2005. Depressive symptoms and carotid artery intima-media thickness in young adults: the Cardiovascular Risk in Young Finns Study. Psychosomatic Medicine, 67, 561-567.

Evren, C., Evren, B., Yancar, C., \& Erkiran, M. (2007). Temperament and character model of personality profile of alcohol-and drug-dependent inpatients. Comprehensive Psychiatry, 48, 283288. https://doi.org/10.1016/j.comppsych.2006.11.003

Farmer, A., Mahmood, A., Redman, K., Harris, T., Sadler, S., \& McGuffin, P. (2003). A sib-pair study of the Temperament and Character Inventory scales in major depression. Archives of General Psychiatry, 60, 490-496. https://doi.org/10.1001/archpsyc.60.5.490

Fils, J. M., Penick, E. C., Nickel, E. J., Othmer, E., DeSouza, C., Gabrielli, W. F., \& Hunter, E. E. (2010). Minor versus major depression: a comparative clinical study. Primary care companion to the Journal of clinical psychiatry, 12(1). https://doi.org/10.4088/PCC.08m00752blu

Fitzgerald, P. B., Laird, A. R., Maller, J., \& Daskalakis, Z. J. (2008). A meta-analytic study of changes in brain activation in depression. Human Brain Mapping, 29, 683-695.

Fried, E. I., \& Nesse, R. M. (2015). Depression is not a consistent syndrome: an investigation of unique symptom patterns in the STAR*D study. Journal of Affective Disorders, 172, 96-102. https://doi.org/10.1016/j.jad.2014.10.010

García, Ó., Aluja, A., García, L. F., Escorial, S., \& Blanch, A. (2012). Zuckerman-Kuhlman-Aluja Personality Questionnaire (ZKA-PQ) and Cloninger's Temperament and Character Inventory 
Revised (TCI-R): A comparative study. Scandinavian Journal of Psychology, 53, 247-257.

https://doi.org/10.1111/j.1467-9450.2012.00943.x

Gilbert, P. (2014). The origins and nature of compassion focused therapy. British Journal of Clinical Psychology, 53, 6-41. DOI:10.1111/bjc.12043

Gilbert P. (2017). Compassion: Concepts, Research and Applications (p. 31-68), edited by P. Gilbert. London: Routledge.

Gilbert, P \& Mascaro, J. (2017). Compassion: Fears, blocks, and resistances: An evolutionary investigation. In, Seppälä, E.M., Simon-Thomas, E., Brown, S.L., Worline, M.C., Cameron, L \& Doty, J. R. (Eds). The Oxford Handbook of Compassion Science. (p.399-420). New York: Oxford University Press.

Gilbert, P. (2015). Affiliative and prosocial motives and emotions in mental health. Dialogues in Clinical Neuroscience, 17, 381-389.

Gilbert, P. (2019). Explorations into the nature and function of compassion. Current Opinion in Psychology, 28, 108-114.

Goldney, R. D., Fisher, L. J., Dal Grande, E., \& Taylor, A. W. (2004). Subsyndromal depression: prevalence, use of health services and quality of life in an Australian population. Social Psychiatry and Psychiatric Epidemiology, 39, 293-298. https://doi.org/10.1007/s00127-004-0745-5

Graser, J., Höfling, V., Weßlau, C., Mendes, A., \& Stangier, U. (2016). Effects of a 12-week mindfulness, compassion, and loving kindness program on chronic depression: A pilot withinsubjects wait-list controlled trial. Journal of Cognitive Psychotherapy, 30, 35-49. https://doi.org/10.1891/0889-8391.30.1.35 
Gilbert, P., \& Procter, S. (2006). Compassionate mind training for people with high shame and selfcriticism: Overview and pilot study of a group therapy approach. Clinical Psychology \& Psychotherapy, 13, 353-379. https://doi.org/10.1002/cpp.507

Gluschkoff, K., Oksman, E., Knafo-Noam, A., Dobewall, H., Hintsa, T., Keltikangas-Järvinen, L., \& Hintsanen, M. (2018). The early roots of compassion: From child care arrangements to dispositional compassion in adulthood. Personality and Individual Differences, 129, 28-32. https://doi.org/10.1016/j.paid.2018.03.005

Gluschkoff, K., Pulkki-Råback, L., Elovainio, M., Saarinen, A., Tammelin, T., Hirvensalo, M., Lehtimäki, T., Keltikangas-Järvinen, L., Raitakari, O., Hintsanen, M. (2018). Is it good to be good? Dispositional compassion and health behaviors. Annals of Behavioral Medicine. https://doi.org/10.1093/abm/kay075

Goetz, J. L., Keltner, D., \& Simon-Thomas, E. (2010). Compassion: an evolutionary analysis and empirical review. Psychological Bulletin, 136, 351-374. http://dx.doi.org/10.1037/a0018807

Gotlib, I. H., \& Joormann, J. (2010). Cognition and depression: current status and future directions. Annual Review of Clinical Psychology, 6, 285-312. https://doi.org/10.1146/annurev.clinpsy.121208.131305

Hansenne, M., Delhez, M., \& Cloninger, C. R. (2005). Psychometric properties of the Temperament and Character Inventory-Revised (TCI-R) in a Belgian sample. Journal of Personality Assessment, 85, 40-49. https://doi.org/10.1207/s15327752jpa8501_04

Hansenne, M., Reggers, J., Pinto, E., Kjiri, K., Ajamier, A., \& Ansseau, M. (1999). Temperament and character inventory (TCI) and depression. Journal of Psychiatric Research, 33, 31-36. https://doi.org/10.1016/S0022-3956(98)00036-3 
Harald, B., \& Gordon, P. (2012). Meta-review of depressive subtyping models. Journal of Affective Disorders, 139, 126-140.

Hu, L. T., \& Bentler, P. M. (1999). Cutoff criteria for fit indexes in covariance structure analysis:

Conventional criteria versus new alternatives. Structural Equation Modeling: A Multidisciplinary Journal, 6, 1-55. https://doi.org/10.1080/10705519909540118

Hur, J. W., \& Kim, Y. K. (2009). Comparison of clinical features and personality dimensions between patients with major depressive disorder and normal control. Psychiatry Investigation, 6, 150-155. https://doi.org/10.4306/pi.2009.6.3.150

Hutcherson, C. A., Seppala, E. M., \& Gross, J. J. (2008). Loving-kindness meditation increases social connectedness. Emotion, 8, 720 .

Immordino-Yang, M. H., McColl, A., Damasio, H., \& Damasio, A. (2009). Neural correlates of admiration and compassion. Proceedings of the National Academy of Sciences, 106, 8021-8026. https://doi.org/10.1073pnas.0810363106

Judge, L., Cleghorn, A., McEwan, K., \& Gilbert, P. (2012). An exploration of group-based compassion focused therapy for a heterogeneous range of clients presenting to a community mental health team. International Journal of Cognitive Therapy, 5, 420-429. https://doi.org/10.1521/ijct.2012.5.4.420

Katainen, S., Räikkönen, K., \& Keltikangas-Järvinen, L. (1999). Adolescent temperament, perceived social support, and depressive tendencies as predictors of depressive tendencies in young adulthood. European Journal of Personality, 13, 183-207. https://doi.org/10.1002/(SICI)10990984(199905/06)13:3<183::AID-PER327>3.0.CO;2-Z

Kirby, J \& Gilbert, P. (2017). The emergence of the Compassion Focused Therapies. In: P. Gilbert (Ed). Compassion: Concepts, Research and Applications (p. 258-285). London: Routledge. 
Klimecki, O. M., Leiberg, S., Ricard, M., \& Singer, T. (2013). Differential pattern of functional brain plasticity after compassion and empathy training. Social Cognitive and Affective Neuroscience, 9, 873-879. https://doi.org/10.1093/scan/nst060

Kogan, A., Saslow, L. R., Impett, E. A., Oveis, C., Keltner, D., \& Saturn, S. R. (2011). Thin-slicing study of the oxytocin receptor (OXTR) gene and the evaluation and expression of the prosocial disposition. Proceedings of the National Academy of Sciences, 108, 19189-19192.

Leaviss, J., \& Uttley, L. (2015). Psychotherapeutic benefits of compassion-focused therapy: An early systematic review. Psychological Medicine, 45, 927-945.

https://doi.org/10.1017/S0033291714002141

Lee, J., Min, S. K., Kim, K.-H., Kim, B., Cho, S. J., Lee, S.-H., . . Suh, S.-Y. (2012). Differences in temperament and character dimensions of personality between patients with Hwa-byung, an anger syndrome, and patients with major depressive disorder. Journal of Affective Disorders, 138, 110-116. https://doi.org/10.1016/j.jad.2011.12.014

Lee, Y. Y., Stockings, E. A., Harris, M. G., Doi, S. A. R., Page, I. S., Davidson, S. K., \& Barendregt, J. J. (2018). The risk of developing major depression among individuals with subthreshold depression: a systematic review and meta-analysis of longitudinal cohort studies. Psychological Medicine, 13, $1-11$. https://doi.org/10.1017/S0033291718000557

Lim, D. \& DeSteno, D. (2016). Suffering and compassion: The links among adverse life experiences, empahty, compassion, and prosocial behavior. Emotion, 16, 175-182.

https://doi.org/10.1037/emo0000144 
Liotti, G., \& Gilbert, P. (2011). Mentalizing, motivation, and social mentalities: Theoretical considerations and implications for psychotherapy. Psychology and Psychotherapy: Theory, Research and Practice, 84, 9-25.

Lucre, K. M., \& Corten, N. (2013). An exploration of group compassion-focused therapy for personality disorder. Psychology and Psychotherapy: Theory, Research and Practice, 86, 387-400. https://doi.org/10.1111/j.2044-8341.2012.02068.x

McEwan, K., Gilbert, P \& Duarte, J. (2012). An exploration of competitiveness and caring in relation to psychopathology. British Journal of Clinical Psychology 51, 19-36.

https://doi.org/10.1111/j.2044-8260.2011.02010.x

McQuaid, R. J., McInnis, O. A., Abizaid, A., \& Anisman, H. (2014). Making room for oxytocin in understanding depression. Neuroscience \& Biobehavioral Reviews, 45, 305-322.

Nurmi, J. E., Salmela-Aro, K., \& Haavisto, T. (1995). The Strategy and Attribution Questionnaire: Psychometric Properties. European Journal of Psychological Assessment, 11, 108-121. https://doi.org/10.1027/1015-5759.11.2.108

O’Connor, L. E., Berry, J. W., Weiss, J., \& Gilbert, P. (2002). Guilt, fear, submission, and empathy in depression. Journal of Affective Disorders, 71, 19-27. https://doi.org/10.1016/S0165$\underline{0327(01) 00408-6}$

Paal, T., \& Bereczkei, T. (2007). Adult theory of mind, cooperation, Machiavellianism: The effect of mindreading on social relations. Personality and Individual differences, 43, 541-551. https://doi.org/10.1016/j.paid.2006.12.021

Pace, T. W., Negi, L. T., Adame, D. D., Cole, S. P., Sivilli, T. I., Brown, T. D., ... \& Raison, C. L. (2009). Effect of compassion meditation on neuroendocrine, innate immune and behavioral 
responses to psychosocial stress. Psychoneuroendocrinology, 34, 87-98.

https://doi.org/10.1016/j.psyneuen.2008.08.011

Pulkki-Råback, L., Elovainio, M., Hakulinen, C., Lipsanen, J., Hintsanen, M., Jokela, M., ... \& Keltikangas-Järvinen, L. (2015). Cumulative effect of psychosocial factors in youth on ideal cardiovascular health in adulthood: the Cardiovascular Risk in Young Finns Study. Circulation, 113.

Raitakari, O. T., Juonala, M., Rönnemaa, T., Keltikangas-Järvinen, L., Räsänen, L., Pietikäinen, M., ..., Jula, A. (2008). Cohort profile: the cardiovascular risk in Young Finns Study. International Journal of Epidemiology, 37, 1220-1226. https://doi.org/10.1093/ije/dym225

Rantala, M. J., Luoto, S., Krams, I., \& Karlsson, H. (2017). Depression subtyping based on evolutionary psychiatry: proximate mechanisms and ultimate functions. Brain, Behavior, and Immunity, 69, 603-617. https://doi.org/10.1016/j.bbi.2017.10.012

Richter, J., Eisemann, M., \& Richter, G. (2000). Temperament and character during the course of unipolar depression among inpatients. European Archives of Psychiatry and Clinical Neuroscience, 250, 40-47. https://doi.org/10.1007/PL00007538

Roisman, G. I., Masten, A. S., Coatsworth, J. D., \& Tellegen, A. (2004). Salient and emerging developmental tasks in the transition to adulthood. Child Development, 75, 123-133. https://doi.org/10.1111/j.1467-8624.2004.00658.x

Rosenström, T., Jokela, M., Puttonen, S., Hintsanen, M., Pulkki-Råback, L., Viikari, J.S., ..., Keltikangas-Järvinen, L. (2012). Pairwise Measures of Causal Direction in the Epidemiology of Sleep Problems and Depression. PLoS One, 7, e50841. https://doi.org/10.1371/journal.pone.0050841 Schiep, S., \& Cieślik, K. (2011). Personality dimensions and nicotine dependence and withdrawal symptoms: the mediating role of self-directness. Polish Psychological Bulletin, 42, 169-177. https://doi.org/10.2478/v10059-011-0022-x 
Schreiber, J. B., Nora, A., Stage, F. K., Barlow, E. A., \& King, J. (2006). Reporting structural equation modeling and confirmatory factor analysis results: A review. The Journal of Educational Research, 99, 323-338. https://doi.org/10.3200/JOER.99.6.323-338

Schreiter, S., Pijnenborg, G. H. M., \& Aan Het Rot, M. (2013). Empathy in adults with clinical or subclinical depressive symptoms. Journal of Affective Disorders, 150, 1-16. https://doi.org/10.1016/j.jad.2013.03.009

Schmidt, K. M., Llewellyn, P. L., Taylor, G. J., Weber, P. G., Hong, B., Sellers, R., Wise, C., Wolak, C., McGaw, L., \& Nielson, S. (2003). Cloninger's Temperament and Character Inventory Correlates with Personality Characteristics of Organ Donation Advocates. Journal of Clinical Psychology in Medical Settings, 10, 173-185. https://doi.org/10.1023/A:1025458728151

Seppälä, E.M., Simon-Thomas, S., Brown, S.L., Worline, M.C., Cameron, C.D \& Doty, J.R (2017, eds.) The Oxford Handbook of compassion science. New York. Oxford University Press.

Sheldon, K. M., \& Kasser, T. (2001). Getting older, getting better? Personal strivings and psychological maturity across the life span. Developmental psychology, 37(4), 491.

Singer, T., \& Klimecki, O. M. (2014). Empathy and compassion. Current Biology, 24, 875-878. https://doi.org/10.1016/j.cub.2014.06.054

Stellar, J. E., Cohen, A., Oveis, C., \& Keltner, D. (2015). Affective and physiological responses to the suffering of others: Compassion and vagal activity. Journal of Personality and Social Psychology, 108, 572-585. http://dx.doi.org/10.1037/pspi0000010

Thayer, J. F., Hansen, A. L., Saus-Rose, E., \& Johnsen, B. H. (2009). Heart rate variability, prefrontal neural function, and cognitive performance: the neurovisceral integration perspective on selfregulation, adaptation, and health. Annals of Behavioral Medicine, 37, 141-153. 
Tully, E. C., Ames, A. M., Garcia, S. E., \& Donohue, M. R. (2016). Quadratic associations between empathy and depression as moderated by emotion dysregulation. The Journal of Psychology, 150, 15-35. https://doi.org/10.1080/00223980.2014.992382

Valk, S. L., Bernhardt, B. C., Trautwein, F. M., Böckler, A., Kanske, P., Guizard, N., Collins, D.L \& Singer, T. (2017). Structural plasticity of the social brain: Differential change after socio-affective and cognitive mental training. Science Advances, 3, e1700489.

Van den Biggelaar, A. H., Gussekloo, J., de Craen, A. J., Frölich, M., Stek, M. L., van der Mast, R. C., \& Westendorp, R. G. (2007). Inflammation and interleukin-1 signaling network contribute to depressive symptoms but not cognitive decline in old age. Experimental Gerontology, 42, 693-701. https://doi.org/10.1016/j.exger.2007.01.011

Van Zoonen, K., Buntrock, C., Ebert, D. D., Smit, F., Reynolds III, C. F., Beekman, A. T., \& Cuijpers, P. (2014). Preventing the onset of major depressive disorder: a meta-analytic review of psychological interventions. International Journal of Epidemiology, 43, 318-329. https://doi.org/10.1093/ije/dyt175

Vervaet, M., Van Heeringen, C., \& Audenaert, K. (2004). Personality-related characteristics in restricting versus binging and purging eating disordered patients. Comprehensive Psychiatry, 45, 37-43. https://doi.org/10.1016/j.comppsych.2003.09.008

Weng, H. Y., Fox, A. S., Shackman, A. J., Stodola, D. E., Caldwell, J. Z., Olson, M. C., . . Davidson, R. J. (2013). Compassion training alters altruism and neural responses to suffering. Psychological Science, 24, 1171-1180.

Weng, H. Y., Lapate, R. C., Stodola, D. E., Rogers, G. M., \& Davidson, R. J. (2018). Visual attention to suffering after compassion training is associated with decreased amygdala responses. Frontiers in Psychology, 9 . 
Wink, P., \& Dillon, M. (2002). Spiritual development across the adult life course: Findings from a longitudinal study. Journal of Adult Development, 9, 79-94.

https://doi.org/10.1023/A:1013833419122 
Table 1. The means, standard deviations (SD), and frequencies of the study variables.

\begin{tabular}{llll}
\hline & Mean / Frequency (\%) & SD & Range \\
\hline Dispositional compassion in 1997 & 3.62 & 0.67 & $1-5$ \\
& & & \\
Total depressive symptoms in 1997 (the mBDI) & 2.10 & 0.65 & $1-5$ \\
Depressive subsymptoms (the mBDI) & & & \\
$\quad$ Negative attitude & 2.19 & 0.70 & $1-5$ \\
Performance difficulties & 2.07 & 0.76 & $1-5$ \\
Somatic complaints & 2.03 & 0.71 & $1-5$ \\
& & & \\
Gender (Female) & $943(56.3)$ & 5.01 & $20-35$ \\
Age in 1997 & 27.69 & & \\
Parental educational level & & & \\
Comprehensive school (the first 9 years of school) & $542(32.3)$ & & \\
High school or occupational school & $692(41.3)$ & & \\
Academic level (university or college) & $442(26.4)$ & 1.92 & $8-34$ \\
Parents' annual income & 4.91 & 3.59 & $1-13$ \\
Participants number of educational years & 15.53 & 3.08 & \\
Participants' annual income & 7.38 & & \\
\hline
\end{tabular}


Table 2. The goodness-of-fit statistics for the longitudinal models on the predictive relationships of compassion with depressive symptoms.

\begin{tabular}{llllllllll}
\hline & & & & & & & \multicolumn{3}{c}{ Model comparisons } \\
\cline { 7 - 10 } & $\chi^{2}$ value & $d f$ & $p$ & RMSEA & CFI & BIC & $\chi^{2}$ difference test & $d f$ & $p$ \\
\hline Model 1 & 552.361 & 88 & $<.001$ & 0.056 & 0.959 & 64308.345 & & & \\
Model 2 & 506.453 & 82 & $<.001$ & 0.056 & 0.963 & 64306.981 & $\chi^{2}(2$ vs. 1) $=45.908$ & 6 & $<.001$ \\
Model 3 & 546.198 & 82 & $<.001$ & 0.058 & 0.959 & 64346.727 & $\chi^{2}(3$ vs. 1)=6.163 & 6 & 0.405 \\
Model 4 & 499.917 & 76 & $<.001$ & 0.058 & 0.963 & 64344.991 & $\chi^{2}(4$ vs. 2)=6.536 & 6 & 0.366 \\
\hline
\end{tabular}

RMSEA $=$ the Root Mean Square Error of Approximation. CFI $=$ the Comparative Fit Index.

$\mathrm{BIC}=$ the Bayesian Information Criterion. $N=1676$

Model 1: Autoregressive model (no cross-lagged predictive paths).

Model 2: Cross-lagged predictive paths from dispositional compassion to depressive symptoms.

Model 3: Cross-lagged predictive paths from depressive symptoms to dispositional compassion.

Model 4: Cross-lagged predictive paths in both directions. 
Dispositional compassion and depressive symptoms

Table 3. Results of multilevel models with longitudinal design. Estimates ( $\beta$ ) with $95 \%$ confidence intervals (CI) of compassion and age, when predicting standardized scores of depressive symptoms.

\begin{tabular}{|c|c|c|c|c|c|c|c|c|}
\hline & \multicolumn{6}{|c|}{ Depressive subsymptoms } & & \\
\hline & \multicolumn{2}{|c|}{ Negative attitude } & \multicolumn{2}{|c|}{ Performance difficulties } & \multicolumn{2}{|c|}{ Somatic complaints } & \multicolumn{2}{|c|}{ Total depressive symptoms } \\
\hline & $\beta$ & $95 \% \mathrm{CI}$ & $\beta$ & $95 \% \mathrm{CI}$ & $\beta$ & $95 \% \mathrm{CI}$ & $\beta$ & $95 \% \mathrm{CI}$ \\
\hline \multicolumn{9}{|l|}{ Fixed effects } \\
\hline Intercept & 0.14 & $-0.0056 ; 0.29$ & 0.064 & $-0.084 ; 0.21$ & -0.021 & $-0.17 ; 0.13$ & 0.084 & $-0.065 ; 0.23$ \\
\hline Compassion & $-0.29 * * *$ & $-0.36 ;-0.22$ & $-0.31 * * *$ & $-0.39 ;-0.24$ & $-0.24 * * *$ & $-0.33 ;-0.17$ & $-0.32 * * *$ & $-0.39 ;-0.25$ \\
\hline Age & $-0.024 * * *$ & $-0.035 ;-0.014$ & $-0.018 * * *$ & $-0.029 ;-0.0081$ & $0.024 * * *$ & $0.013 ; 0.035$ & -0.0099 & $-0.020 ; 0.00015$ \\
\hline Age squared & $0.00040 *$ & $0.000074 ; 0.00072$ & $0.00043 *$ & $0.00011 ; 0.00075$ & -0.00025 & $-0.00059 ; 0.000099$ & 0.00026 & $-0.000055 ; 0.00057$ \\
\hline $\begin{array}{l}\text { Compassion* } \\
\text { Age }\end{array}$ & 0.0042 & $-0.0061 ; 0.015$ & 0.0077 & $-0.0026 ; 0.018$ & 0.0083 & $-0.0027 ; 0.019$ & 0.0076 & $-0.0024 ; 0.018$ \\
\hline $\begin{array}{l}\text { Compassion* } \\
\text { Age squared }\end{array}$ & -0.00025 & $-0.00059 ; 0.000094$ & $-0.00036^{*}$ & $-0.00070 ;-0.000026$ & $-0.00038^{*}$ & $-0.00075 ;-0.000017$ & $-0.00037 *$ & $-0.00070 ;-0.000046$ \\
\hline \multicolumn{9}{|l|}{ Random effects } \\
\hline Variance of intercept & $0.76^{*}$ & $0.70 ; 0.83$ & $0.71 *$ & $0.65 ; 0.79$ & $0.66^{*}$ & $0.58 ; 0.75$ & $0.74 *$ & $0.68 ; 0.81$ \\
\hline Variance of age & $0.028 *$ & $0.023 ; 0.035$ & $0.020 *$ & $0.013 ; 0.030$ & $0.021 *$ & $0.013 ; 0.033$ & $0.026^{*}$ & $0.020 ; 0.33$ \\
\hline Residual variance & $0.60 *$ & $0.58 ; 0.63$ & $0.63 *$ & $0.60 ; 0.65$ & $0.69 *$ & $0.66 ; 0.72$ & $0.59 *$ & $0.57 ; 0.61$ \\
\hline
\end{tabular}




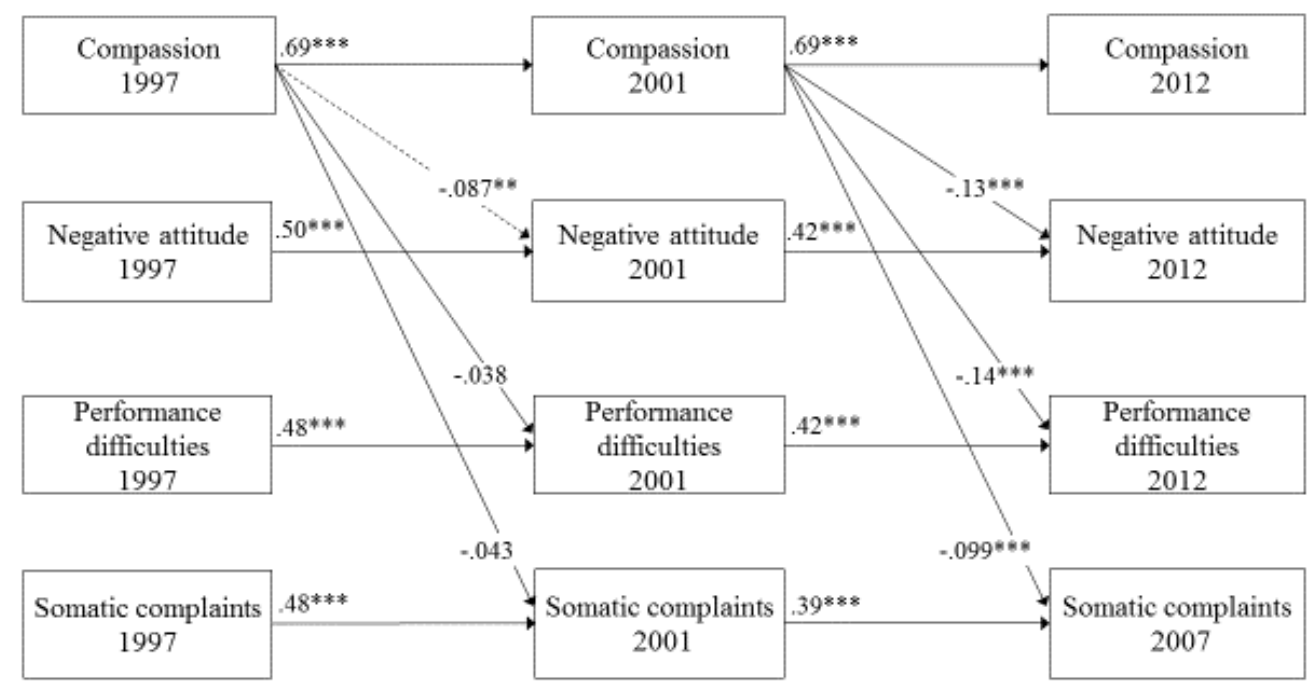

Figure 1. Standardized regression paths of the model 2 (i.e. stability coefficients and the cross-lagged paths from dispositional compassion depressive symptoms). Note: For clarity, control variables (age, gender, socioeconomic factors in childhood and adulthood) and the covariances between variables at each time point are not shown in the figure. ${ }^{* * *} \mathrm{p}<.001 * * \mathrm{p}<.01 \mathrm{~N}=1676$ 
(a) Total depressive symptoms.

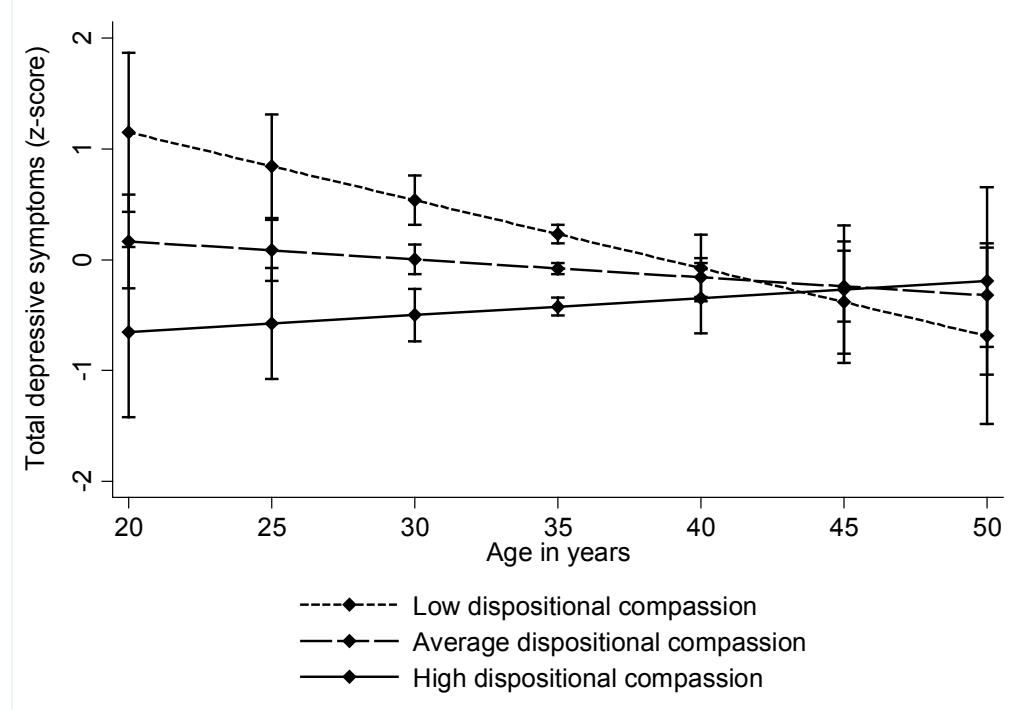

(b) Depression-related negative attitude.

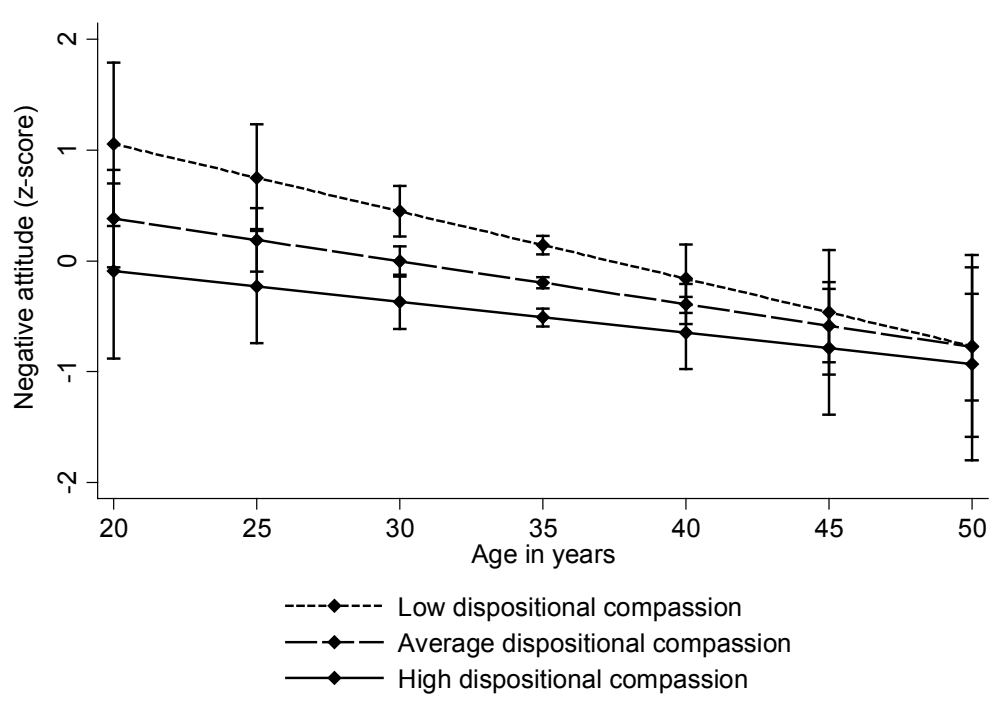

(c) Depression-related performance difficulties. 


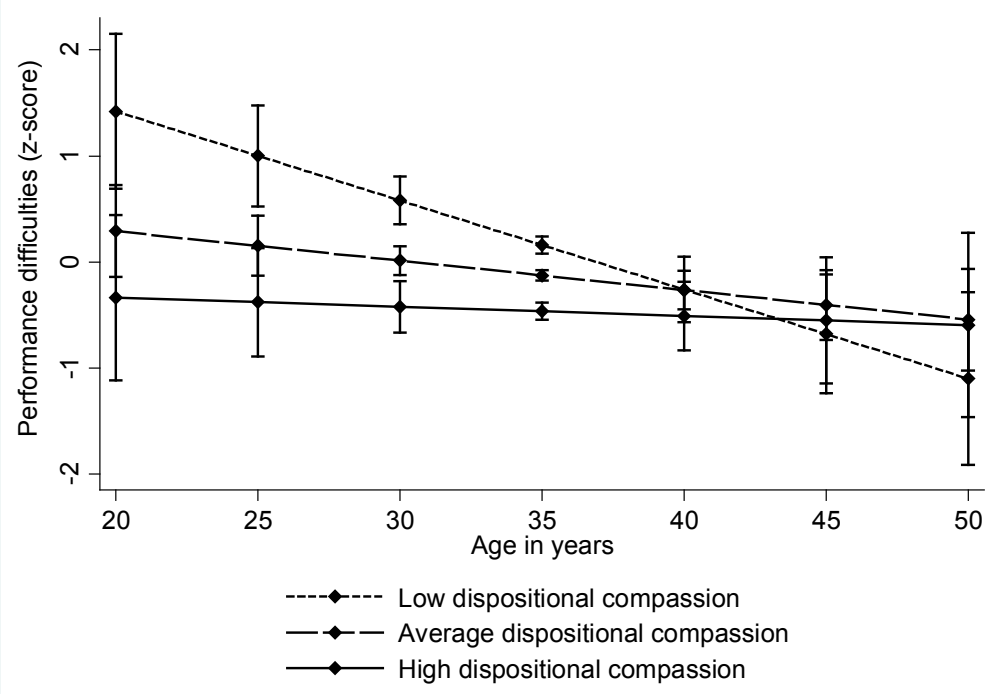

(d) Depression-related somatic complaints.

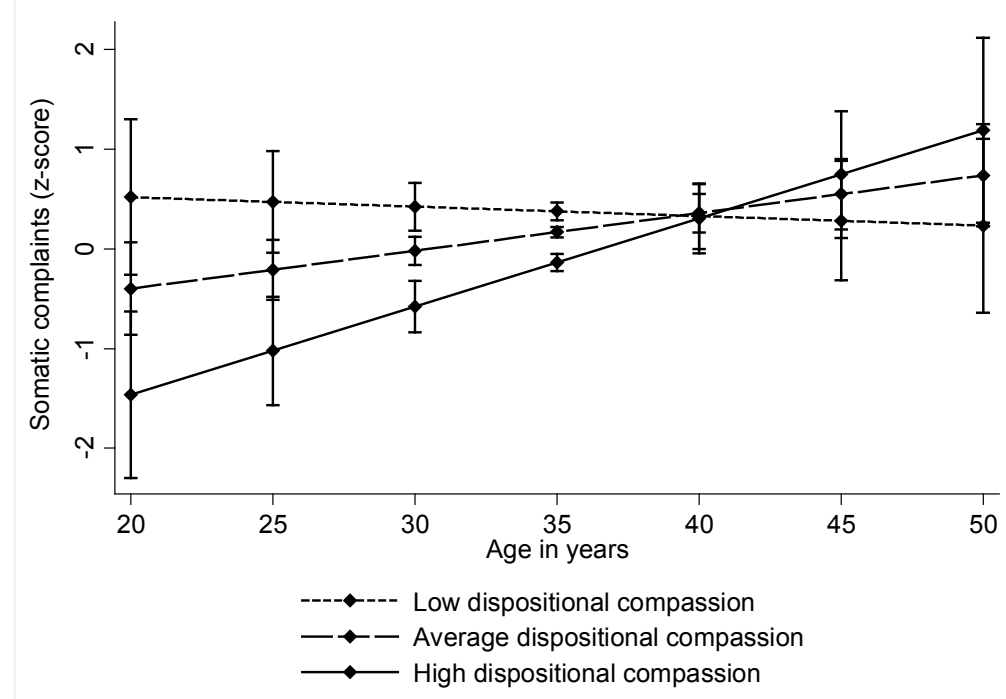

Figures 2a-d. Growth trajectories of standardized scores of (a) total score of depressive symptoms, (b) negative attitude, (c) performance difficulties, and (d) somatic complaints separately for participants belonging to the lowest $20 \%$, average, and the highest $20 \%$ in dispositional compassion for others. Predicted means with 95\% confidence intervals. 
Dispositional compassion and depressive symptoms 